\title{
Superconducting Interfaces between Insulating Oxides
}

\author{
N. Reyren ${ }^{1}$, S. Thiel ${ }^{2}$, A. D. Caviglia ${ }^{1}$, L. Fitting Kourkoutis ${ }^{3}$, \\ G. Hammerl ${ }^{2}$, C. Richter ${ }^{2}$, C. W. Schneider ${ }^{2}$, T. Kopp ${ }^{2}$, A.-S. Ruetschi ${ }^{1}$, \\ D. Jaccard ${ }^{1}$, M. Gabay ${ }^{4}$, D. A. Muller ${ }^{3}$, J.-M. Triscone ${ }^{1}$, J. Mannhart ${ }^{2}$
}

${ }^{1}$ DPMC, University of Geneva, 24 quai Ernest-Ansermet, 1211 Genève 4, Switzerland

${ }^{2}$ Experimental Physics VI, Center for Electronic Correlations and Magnetism, Institute of Physics, University of Augsburg, D-86135 Augsburg, Germany

${ }^{3}$ School of Applied and Engineering Physics, Cornell University, Ithaca, NY 14853, USA

${ }^{4}$ Laboratoire de Physique des Solides, Bat 510

Université Paris-Sud 11, Centre d'Orsay, 91405 Orsay, Cedex, France

*To whom the correspondence should be addressed: E-Mail: jochen.mannhart@ physik.uni-augsburg.de

\begin{abstract}
At interfaces between complex oxides, electronic systems with unusual electronic properties can be generated. Here, we report on superconductivity in the electron gas formed at the interface between the insulators $\mathrm{LaAlO}_{3}$ and $\mathrm{SrTiO}_{3}$. The behavior of the electron gas is that of a two dimensional superconductor, confined to a thin sheet at the interface. The superconducting transition temperature of $\simeq 200 \mathrm{mK}$ provides a strict upper limit to the thickness of the superconducting layer of $\simeq 10 \mathrm{~nm}$.
\end{abstract}


In a pioneering work, Ohtomo and Hwang have demonstrated that a highly mobile electron system can be induced at the interface between $\mathrm{LaAlO}_{3} /$ and $\mathrm{SrTiO}_{3}[1]$. The discovery of this electron gas at the interface between two insulators has generated an impressive amount of experimental and theoretical work [2-7], in part because the complex ionic structure and particular interactions found at such an interface are being expected to promote novel electronic phases, not stabilized in the bulk $[4,8,9]$. This result also generated an intense debate on the origin of this conducting layer which could be "extrinsic" and due to oxygen vacancies in the $\mathrm{SrTiO}_{3}$ crystal or "intrinsic" and related to the polar nature of the $\mathrm{LaAlO}_{3} /$ structure which produces a potential developing as the $\mathrm{LaAlO}_{3} /$ layer thickness increases that may lead to an "electronic reconstruction" above some critical thickness [5, 10]. Another key issue concerns the ground state of such a system; at low temperatures a charge-ordered interface with ferromagnetic spin alignment was predicted [11]. Experimental evidence in favor of a ferromagnetic ground state was recently found [7]. Yet, rather than ordering magnetically, the electron system may also condense into a superconducting state. Already in 1986, it was proposed that in field effect transistor configurations a superconducting, two-dimensional (2D) electron gas might be generated at the $\mathrm{SrTiO}_{3}$ surface [12]. It was also pointed out that[13] the polarization of the $\mathrm{SrTiO}_{3}$ layers may cause the electrons on $\mathrm{SrTiO}_{3}$ surfaces to pair and form at high temperatures a superconducting condensate. In this report, we explore the ground state of the $\mathrm{LaAlO}_{3} / \mathrm{SrTiO}_{3}$ interface and clarify whether it orders when the temperature approaches absolute zero. Our experiments provide evidence that the investigated electron gases condense into a superconducting phase. The characteristics of the transition are consistent with those of a 2D electron system undergoing a Berezinskii-Kosterlitz-Thouless transition [14]. In the oxygen vacancy scenario the observation of superconductivity provides a strict upper limit to the thickness of the superconducting sheet at the $\mathrm{LaAlO}_{3} / \mathrm{SrTiO}_{3}$ interface.

The samples were prepared by depositing $\mathrm{LaAlO}_{3}$ layers with thicknesses of 2, 8, and 15 unit cells (uc) on $\mathrm{TiO}_{2}$-terminated (001) surfaces of $1 \mathrm{~mm}$ thick $\mathrm{SrTiO}_{3}$ single crystals as described in [5]. The $\mathrm{LaAlO}_{3}$ films were grown from a stoichiometric, single crystalline target by pulsed laser deposition at $770^{\circ} \mathrm{C}$ and $6 \times 10^{-5} \mathrm{mbar}_{2}$ at a fluence of $\simeq 1 \mathrm{~J} / \mathrm{cm}^{2}$. One pulse/sec leads to a growth rate of $0.15 \AA / \mathrm{sec}$. After deposition, the samples were cooled to room temperature in $400 \mathrm{mbar}$ of $\mathrm{O}_{2}$. The 2.5 hour cool down included a 1 hour oxidation step at $600^{\circ} \mathrm{C}$. The thickness of the $\mathrm{LaAlO}_{3}$ was controlled at the unit cell level by using a reflection high-energy electron diffraction (RHEED) system that can operate at high oxygen pressures. Because only heterostructures with a $\mathrm{LaAlO}_{3}$ thickness greater than three unit cells conduct [5], samples were 
patterned into conducting and insulating areas [15]. Without exposing the $\mathrm{LaAlO}_{3} / \mathrm{SrTiO}_{3}$ interface to chemicals or to the environment, bridges with widths of $100 \mu \mathrm{m}$ and lengths of $300 \mu \mathrm{m}$ and $700 \mu \mathrm{m}$ were structured for four-point measurements. On each sample control areas with two unit cell thick $\mathrm{LaAlO}_{3}$ layers were patterned. Contacts were provided by Au-filled, square holes with a side length of about $200 \mu \mathrm{m}$ that were ion milled through the electron gas [15]. To avoid photoconduction all transport measurements were done after keeping the samples in the dark for at least 20 hours.

Transmission electron studies were performed on reference samples grown under conditions identical to those described above. Cross-sectional cuts were prepared by mechanical polishing followed by low-energy, low-angle ion milling and investigated by scanning transmission electron microscopy (STEM) [16]. Fig. 1A shows a high angle annular dark field (HAADF) STEM image of the sharp interface between a 15 uc thick $\mathrm{LaAlO}_{3}$ film and the $\mathrm{SrTiO}_{3}$ substrate. The film is found to be commensurate (xxx: Dave: ok?) with the substrate with no obvious defects or dislocations at the interface, resulting in biaxial tensile strain of $\simeq 3 \%$, as measured from STEM images. The out-of-plane lattice constant of the $\mathrm{LaAlO}_{3}$ film is $\simeq 3.78 \AA$ which is close to the bulk value and suggests either a rather small Poisson ratio as previously reported [17] or out-ofplane relaxation in the thin film [18]. To obtain an upper limit on the extent of electronic structure and compositional changes below the interface, electron energy loss spectroscopy (EELS) in the STEM was used to probe the chemistry of the heterostructure at the atomic scale. Simultaneously recorded $\mathrm{O}-\mathrm{K}$ and $\mathrm{Ti}-\mathrm{L}_{2,3}$ edges close to and far away from the interface are shown in Fig. 1B and $1 \mathrm{C}$. By $1.5 \mathrm{~nm}$ away from the interface, the changes in the $\mathrm{O}-\mathrm{K}$ edge are very slight, suggesting an upper limit to the oxygen vacancy concentration of $3 \%$. At $6 \mathrm{~nm}$ away from the interface the changes in the $\mathrm{O}-\mathrm{K}$ and $\mathrm{Ti}-\mathrm{L}_{2,3}$ edges compared to bulk $\mathrm{SrTiO}_{3}$ fall below the noise level $(<1 \%$ oxygen vacancy concentration). The small changes of the Ti- $\mathrm{L}_{2,3}$ edges are consistent with a slight increase in $\mathrm{Ti}^{3+}$, either from oxygen deficiency or a compensating interface charge.

The HAADF STEM image (Fig. 1A) reveals a dark band in the $\mathrm{SrTiO}_{3}$ close to the $\mathrm{LaAlO}_{3} /$ $\mathrm{SrTiO}_{3}$ interface. The reduced HAADF intensity at the interface has to be interpreted with care. Bending/tilting of the crystal in that region or distortions of the thin TEM foil due to strain relaxation can result in a reduction of the HAADF intensity [19], and we do detect a local tilting of $5-10 \mathrm{mrad}$ of the foil in the thinner regions [17]. This buckling of the sample can lead to a dark band at the interface due to dechanneling of the electron beam [19]. While the presence of oxygen vacancies in $\mathrm{SrTiO}_{3}$ can also lead to a reduced HAADF intensity [20], the contrast at low vacancy 
concentrations is expected to be patchier than found here, as we would be observing single vacancies. Furthermore, a band of oxygen deficient $\mathrm{SrTiO}_{3}$ would cause an increase in the low angle ADF STEM signal in that region which was not observed. From this we conclude that the dominant contribution to the observed dark band is the local tilting of the film, not oxygen vacancies, whose signal would be hidden beneath this much larger contribution.

Two samples were analyzed by transport measurements and found to be conducting (Fig. 2A), their 2 uc thick control structures being insulating (resistance $R>30 \mathrm{M} \Omega$ ) at all temperatures $T$ $(32 \mathrm{mK}<T<300 \mathrm{~K})$. At $T \simeq 4.2 \mathrm{~K}$, the Hall carrier densities of the 8 uc and $15 \mathrm{uc}$ samples equal $\simeq 4 \times 10^{13} / \mathrm{cm}^{2}$ and $\simeq 1.5 \times 10^{13} / \mathrm{cm}^{2}$, and the mobilities $\simeq 350 \mathrm{~cm}^{2} / \mathrm{Vs}$ and $\simeq 1000 \mathrm{~cm}^{2} / \mathrm{Vs}$, respectively. Whether the differences of the sample properties present an intrinsic effect that is caused by the variation of the $\mathrm{LaAlO}_{3}$ thickness remains to be explored. The Hall response is only weakly temperature dependent (Hall resistance $R_{\mathrm{H}}(300 \mathrm{~K}) / R_{\mathrm{H}}(4.2 \mathrm{~K}) \simeq 0.8$ and 0.95 for the $8 \mathrm{uc}$ and $15 \mathrm{uc}$ samples). Magnetic fields up to $\mu_{0} H=8 \mathrm{~T}$ were applied to the $8 \mathrm{uc}$ thick sample revealing a positive magnetoresistance. The samples investigated here do not show a hysteretic magnetoresistance. No minimum is found in the $R(T)$ characteristics of the 8 uc sample [21], such as was reported recently for $\mathrm{LaAlO}_{3} / \mathrm{SrTiO}_{3}$ samples fabricated under different conditions [7].

At $\simeq 200 \mathrm{mK}$ and $\simeq 100 \mathrm{mK}$ respectively, the $8 \mathrm{uc}$ and $15 \mathrm{uc}$ samples undergo a transition into a state for which no resistance could be measured (Fig. 2A). The widths of the transitions (20\%$80 \%$ ) of the $8 \mathrm{uc}$ and $15 \mathrm{uc}$ samples are $\simeq 16 \mathrm{mK}$ and $\simeq 51 \mathrm{mK}$, respectively. The resistance drops by more than three orders of magnitude to below the noise limit of the measurement [16]. Application of a magnetic field $\mu_{0} H=180 \mathrm{mT}$ perpendicular to the interface completely suppresses this zero-resistance state (Fig. 2B). Figure 3A displays the voltage versus current $(V(I))$ characteristics of a bridge in the 8 uc sample, measured using a dc technique. At low temperatures, the $V(I)$ characteristics show a well-defined critical current $I_{\mathrm{c}}$. The occurrence of the zero-resistance state and the characteristic $R(T)$ and $V(I, H)$ dependences provide clear evidence for superconductivity.

The $T_{\mathrm{c}}(H)$ dependence, $T_{\mathrm{c}}$ being defined as $R\left(T_{\mathrm{c}}\right)=0.5 \times R(1 \mathrm{~K})$, provides a measure for the upper critical field $H_{\mathrm{c} 2}(T)$. The $H_{\mathrm{c} 2}(T)$ curve is shown in Fig. $2 \mathrm{C} ; H_{\mathrm{c} 2}(0 \mathrm{~K}) \simeq 65 \mathrm{mT}$ and $\simeq 30 \mathrm{mT}$ for the $8 \mathrm{uc}$ and $15 \mathrm{uc}$ samples, corresponding to coherence lengths $\xi(0 \mathrm{~K}) \simeq 70 \mathrm{~nm}$ and $\simeq 105 \mathrm{~nm}$. Figure $3 \mathrm{~B}$ shows the temperature dependence of the critical currents per unit width. The maximal values of $I_{\mathrm{c}}$ are $98 \mu \mathrm{A} / \mathrm{cm}$ and $5.6 \mu \mathrm{A} / \mathrm{cm}$ for the $8 \mathrm{uc}$ and $15 \mathrm{uc}$ samples, respectively. A step-like structure in the $V(I)$ curves displayed by the 15 uc sample (not shown) indicates that the low $I_{\mathrm{c}}$ of this sample is caused by inhomogeneities. Just below $I_{\mathfrak{c}}$, the samples develop a small voltage 
drop which is proportional to the current and increases with temperature. As Fig. 4A shows for the 8 uc thick sample, at $30 \mathrm{mK}$ the associated resistance is at least four orders of magnitude smaller than the normal state resistance. With $T$ increasing from $30 \mathrm{mK}$ to $180 \mathrm{mK}$, the resistance grows exponentially from $\simeq 0.1 \Omega$ to $10 \Omega$. Between $180 \mathrm{mK}$ and $T_{\mathrm{c}}$, the step at $I_{\mathrm{c}}$ disappears and powerlaw type $V(I)$ curves are measured.

Is the bulk of the $\mathrm{SrTiO}_{3}$ superconducting or is it only a thin sheet at the interface layer? How thick is the superconducting layer? If the heterostructures were 2D superconductors, the transition into the superconducting state would be a BKT transition, characterized by a transition temperature $T_{\mathrm{BKT}}$ at which vortex-antivortex pairs unbind [22-24]. A simple estimate of $T_{\mathrm{BKT}}$ assuming that the sheet superconducting carrier density equals $4 \times 10^{13} / \mathrm{cm}^{2}$, would suggest that in the samples the BKT and mean field temperatures almost coincide. However, in case of large vortex fugacity, a high density of vortex-antivortex pairs is thermally generated and an ionic like vortex-antivortex crystal is formed [25]. For such a system, the melting of this lattice represents the BKT-transition, which then occurs at lower temperatures. At the BKT-transition, the current-induced Lorentz force causes dislocation-antidislocation pairs to unbind, resulting in a $V \propto I^{a}$ behavior, with $a\left(T_{\mathrm{BKT}}\right)=3$.

The samples indeed show clear signatures of the BKT-behavior, such as a $V \propto I^{a}$ power law dependence (Fig. 4A). As revealed by Fig. 4B, at $T=188 \mathrm{mK}$ the exponent $a$ approaches 3; this temperature is therefore identified as $T_{\mathrm{BKT}}$. The $V(I, T)$ characteristics (Fig. 4A) are very similar to the results of simulations treating finite-size 2D systems [26, 27]. The ohmic regime observed below $T_{\mathrm{BKT}}$ at small currents is expected for finite size samples and agrees quantitatively with an analysis [28, 29] based on [25].

Also the $R(T)$ characteristics are consistent with a BKT-transition, for which close to $T_{\mathrm{BKT}}$ a $R=R_{0} \exp \left(-b t^{-1 / 2}\right)$ dependence is expected [30]. Here, $R_{0}$ and $b$ are material parameters and $t=T / T_{\mathrm{BKT}}-1$. As shown by Fig. $4 \mathrm{C}$, the measured $R(T)$ dependence is consistent with this behavior and yields $T_{\mathrm{BKT}} \simeq 190 \mathrm{mK}$, in agreement with the result of the $a$-exponent analysis. The superconducting transition of the samples is therefore consistent with that of a 2D superconducting film. Hence, the superconducting layer is thinner than $\xi \simeq 70 \mathrm{~nm}$.

Analysis of the superconducting transition temperature provides an independent bound on the layer thickness. If the superconductivity was due to oxygen defects in $\mathrm{SrTiO}_{3-x}$, a carrier density of $\gtrsim 3 \times 10^{19} / \mathrm{cm}^{3}$ would be required for a $T_{\mathrm{c}}$ of $200 \mathrm{mK}$ [31]. The measured sheet carrier densities thus give an upper limit for the thickness of the superconducting sheet of $\simeq 15 \mathrm{~nm}$. Considering that the carrier concentration of the $\mathrm{SrTiO}_{3-x}$ layer cannot be constant but has to follow a profile 
following Poisson's equation as treated with consideration of the field-dependent $\mathrm{SrTiO}_{3}$ susceptibilty [32], one can set an upper limit for the thickness of the superconducting sheet of $\simeq 10 \mathrm{~nm}$ [33], a value much smaller than that suggested in [34, 35] for the thickness of the conducting layer in reduced $\mathrm{LaAlO}_{3} / \mathrm{SrTiO}_{3}$ heterostructures. The carrier density profile at interfaces in oxygendeficient $\mathrm{SrTiO}_{3-x}$ has also been calculated in [34]. As a result of this model, a sheet carrier density $>5 \times 10^{14} / \mathrm{cm}^{2}$ is needed to provide a carrier concentration of $3 \times 10^{19} / \mathrm{cm}^{3}$. Because the sheet carrier densities of our samples equal $1.5-4 \times 10^{13} / \mathrm{cm}^{2}$ only, according to this model the superconductivity of the $\mathrm{LaAlO}_{3} / \mathrm{SrTiO}_{3}$ interface cannot be caused by doped $\mathrm{SrTiO}_{3-x}$ alone.

The experiments presented here do not allow us to determine whether the observed superconductivity is due to a thin doped $\mathrm{SrTiO}_{3}$ sheet or a novel phenomenon occurring at this artificial interface. While the $T_{\mathrm{c}}$ of the heterostructures falls in the transition range of oxygen deficient $\mathrm{SrTiO}_{3-x}$, the transport properties of the samples differ to some extent from the ones of doped $\mathrm{SrTiO}_{3}$. In oxygen deficient $\mathrm{SrTiO}_{3-x}$ and $\mathrm{Nb}$-doped $\mathrm{SrTiO}_{3}$ films, the Hall constant increases below $100 \mathrm{~K}$ [36], as it is almost temperature independent in $\mathrm{LaAlO}_{3} / \mathrm{SrTiO}_{3}$ heterostructures. Also the upper critical field of the heterostructures is an order of magnitude smaller than that of $\mathrm{Nb}$ $\mathrm{SrTiO}_{3}$ with the same $T_{\mathrm{c}}$. Finally, the fact that we observe on the same sample superconducting and insulating behavior depending on the precise $\mathrm{LaAlO}_{3}$ layer thickness is very hard to reconcile with a pure oxygen vacancy-scenario.

[1] A. Ohtomo, D. A. Muller, J. L. Grazul, H. Y. Hwang, Nature 419, 378 (2002); A. Ohtomo, H. Y. Hwang, Nature 427, 423 (2004); corr. Nature 441, 120 (2006).

[2] A. Ohtomo, D. A. Muller, J. L. Grazul, H. Y. Hwang, Nature 419, 378 (2002).

[3] A. Othoma, D. A. Muller, J. L. Grazul, H. Y. Hwang, Nature 419, 378 (2002); A. Ohtomo, H. Y. Hwang, Nature 427, 423 (2004); corr. Nature 441, 120 (2006).

[4] S. Okamoto, J. Millis, Nature 428, 630 (2004).

[5] S. Thiel, G. Hammerl, A. Schmehl, C. W. Schneider, J. Mannhart, Science 313, 1942 (2006).

[6] M. et al.. Huijben, Nat. Mater. 5, 556 (2006).

[7] A. Brinkman, M. Huijben, M. van Zalk, J. Huijben, U. Zeitler, J. C. Maan, W. G. van der Wiel, G. Rijnders, D. H. A. Blank, H. Hilgenkamp, Nat. Mater. 6, 493 (2007).

[8] S. Altieri, L. H. Tjeng, G. A. Sawatzky, Thin Solid Films 9, 400 (2001). 
[9] J. Mannhart, Thin Films and Heterostructures for Oxide Electronics, S. Ogale (ed.), Springer (2005).

[10] N. Nakagawa, H. Y. Hwang, M. D. A., Nat. Mater. 5, 204 (2006).

[11] R. Pentcheva, W. E. Pickett, Phys. Rev. B 74, 035112 (2006).

[12] M. Gurvitch, H. L. Stormer, R. C. Dynes, J. M. Graybeal, D. C. Jacobson, Field effect on superconducting surfaces layers of $\mathrm{SrTiO}_{3}$, Proc. of Materials Research Society Symposium (1986).

[13] V. Koerting, Q. Yuan, P. J. Hirschfeld, T. Kopp, J. Mannhart, Phys. Rev. B 71, 104510 (2005).

[14] V. L. Berezinskii, Zh. Eksp. Teor. Fiz. 61, 1144 (1971); Sov., Phys. JETP 34, 610 (1972); J. M. Kosterlitz, D. J. Thouless, J. Phys. C 5, L124 (1972).

[15] C. W. Schneider, S. Thiel, G. Hammerl, C. Richter, J. Mannhart, Appl. Phys. Lett. 89, 122101 (2006).

[16] Materials and methods are available as supporting material on Science Online.

[17] J.-L. Maurice, C. Carretero, M.-J. Casanove, K. Bouzehouane, S. Guyard, E. Larquet, J.-P. Contour, Phys. Stat. Sol. A 203, 2209 (2006).

[18] M. M. J. Treacey, J. M. Gibson, J. Vac. Sci. Tech. B 4, 1458 (1986).

[19] L. Fitting, S. Thiel, A. Schmehl, J. Mannhart, D. A. Muller, Ultramicroscopy 106, 1053 (2006).

[20] D. A. Muller, N. Nakagawa, A. Ohtomo, J. L. Grazul, H. Y. Hwang, Nature 430, 657 (2004).

[21] For the 15 uc sample, a shallow minimum in the $R(T)$ curve was observed at $4 \mathrm{~K}$.

[22] V. L. Berezinskii, Zh. Eksp. Teor. Fiz. 61, 1144 (1971); Sov., Phys. JETP 34, 610 (1972).

[23] J. M. Kosterlitz, D. J. Thouless, J. Phys. C 5, L124 (1972).

[24] M. R. Beasley, J. E. Mooij, T. P. Orlando, Phys. Rev. Lett. 42, 1165 (1979).

[25] M. Gabay, A. Kapitulnik, Phys. Rev. Lett. 71, 2138 (1993).

[26] H. Weber, M. Wallin, H. J. Jensen, Phys. Rev. B 53, 8566 (1996).

[27] K. Medvedyeva, B. J. Kim, P. Minnhagen, Phys. Rev. B 62, 14531 (2000).

[28] The Lorentz force causes a distortion of the crystal structure. The resulting elastic force, $\left(\phi_{0} I / l\right)^{2} /(2 \mu b)(l$ is the sample width, $\mu$ the shear modulus, $b$ the lattice spacing) can overcome the attraction $\left(4 k T_{\mathrm{BKT}} / r\right)$ within a dislocation-antidislocation pair whose size $r$ is large enough. Free defects cause dissipation and when $r \geq l$, i.e. $I \leq I^{*}$, this regime is ohmic. Near $T_{\mathrm{BKT}}, I^{*} \sim$ $\left(4 k T_{\mathrm{BKT}} / \phi_{0}\right)(2 \pi l / b)^{1 / 2}$. The parameter $b \propto \xi(T)$ decreases with decreasing temperature $\left(b\left(T_{\mathrm{BKT}}\right) \approx\right.$ $0.63 \mu \mathrm{m})$.

[29] These predictions are consistent with the experimental findings displayed in Fig. 4A (for instance, $\left.I_{\exp }^{*}\left(T_{\mathrm{BKT}}\right) \approx 0.3 \mu \mathrm{A}\right)$. By contrast, a standard vortex-antivortex unbinding mechanism would yield a $T$ independent $I^{*}[26,27,30]$ and would predict a value of $I^{*}$ two orders of magnitude smaller than 
$I_{\exp }^{*}$ at $T_{\mathrm{BKT}}$. Note that the defects which become unbound as a result of the applied current can get pinned by the modulation of the lattice spacing due to the shearing effect of the Lorentz force; this effect offers a possible explanation of the steps observed in Fig. 3A.

[30] B. I. Halperin, D. R. Nelson, J. Low Temp. 36, 599 (1979).

[31] C. S. Koonce, M. L. Cohen, J. F. Schooley, W. R. Hosler, E. R. Pfeiffer, Phys. Rev. 163, 380 (1967).

[32] K. Ueno, PhD thesis, University of Tokyo, Field-Effect Transistor Based on Perovskite Oxides (2003).

[33] The proximity effect does not alter this upper limit, because the measured sheet carrier density includes the electrons of the normal-metal layer. Furthermore, to compensate for the resulting $T_{\mathrm{c}}$ suppression of the superconducting layer, the proximity effect requires an enhanced carrier density in the superconductor.

[34] W. Siemons, G. Koster, H. Yamamoto, W. A. Harrison, G. Lucovsky, T. H. Geballe, D. H. A. Blank, M. R. Beasley, Phys. Rev. Lett. 98, 196802 (2007).

[35] G. Herranz, M. Basletić, M. Bibes, C. Carrétéro, E. Tafra, E. Jacquet, K. Bouzehouane, C. Deranlot, A. Hamzić, J.-M. Broto, A. Barthélémy, A. Fert, Phys. Rev. Lett. 98, 216803 (2007).

[36] K. S. Takahashi, M. Gabay, D. Jaccard, K. Shibuya, T. Ohnishi, M. Lippmaa, J.-M. Triscone, Nature 441, 195 (2006).

[37] The authors acknowledge fruitful discussions and interactions with D. Matthey and D. G. Schlom. The work was supported by the Swiss National Science Foundation through the NCCR "Materials with Novel Electronic Properties, MaNEP” and Division II, by the Bundesministerium für Bildung und Forschung via EKM (13N6918), by the Deutsche Forschungsgemeinschaft through SFB 484, by the EU through Nanoxide, by the European Science Foundation through THIOX, and by the National Science Foundation (NSF).

\section{Supporting Online Material}

www.sciencemag.org

Materials and Methods 


\section{Figures}
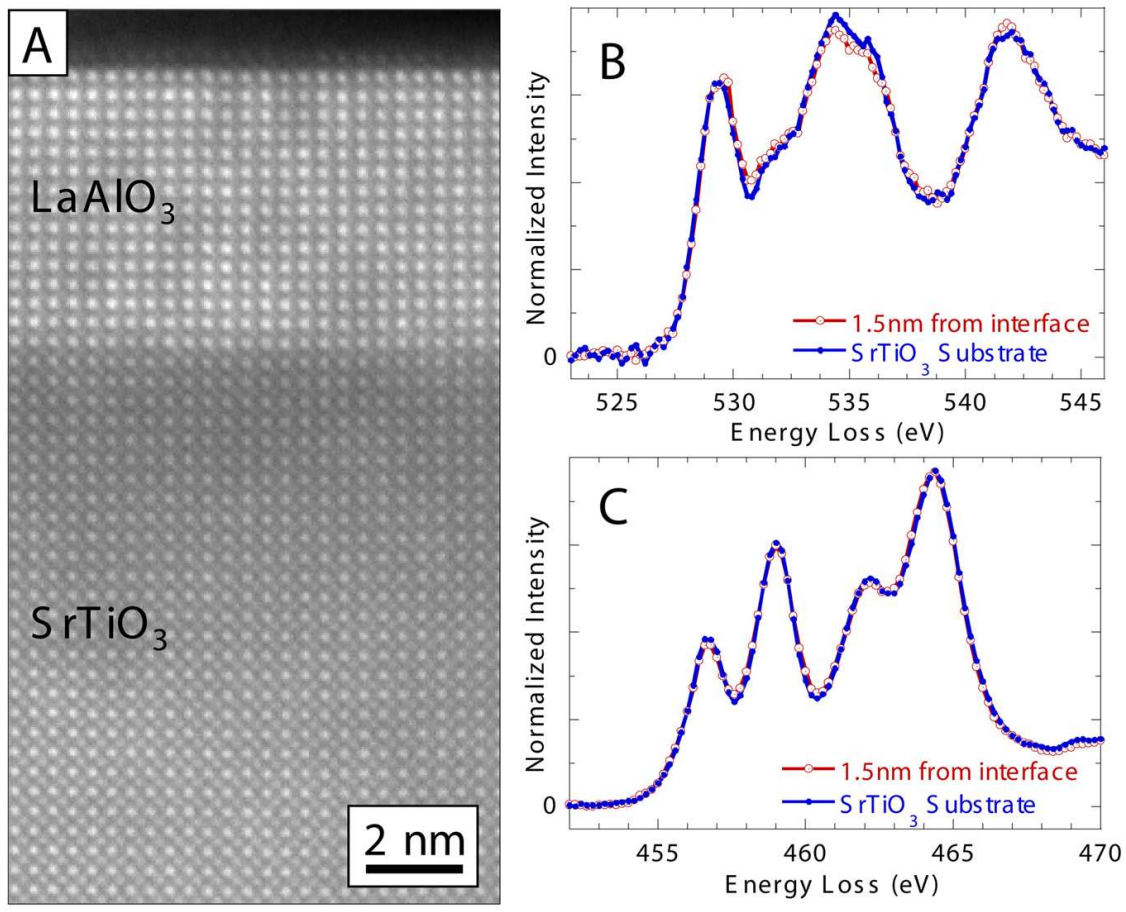

Fig. 1. Scanning transmission electron microscopy (STEM) and electron energy loss spectroscopy (EELS) analysis of a $\mathrm{LaAlO}_{3} / \mathrm{SrTiO}_{3}$ heterostructure. (A) High angle annular dark field image of a 15 uc thick $\mathrm{LaAlO}_{3}$ film grown on $\mathrm{SrTiO}_{3}$ showing a coherent interface. (B) O-K EELS spectra of the $\mathrm{SrTiO}_{3}$ close to $(1.5 \mathrm{~nm})$ and far away from the interface. Even at $1.5 \mathrm{~nm}$ from the interface the $\mathrm{O}-\mathrm{K}$ fine structure is only very slightly damped compared to the bulk. The damping could be caused by the presence of a low concentration of oxygen vacancies. (C) Small changes of the $\mathrm{Ti}-\mathrm{L}_{2,3}$ fine structure close to the interface are consistent with a small concentration of $\mathrm{Ti}^{3+}$, which falls below the detection limit by $6 \mathrm{~nm}$ from the interface and beyond. 

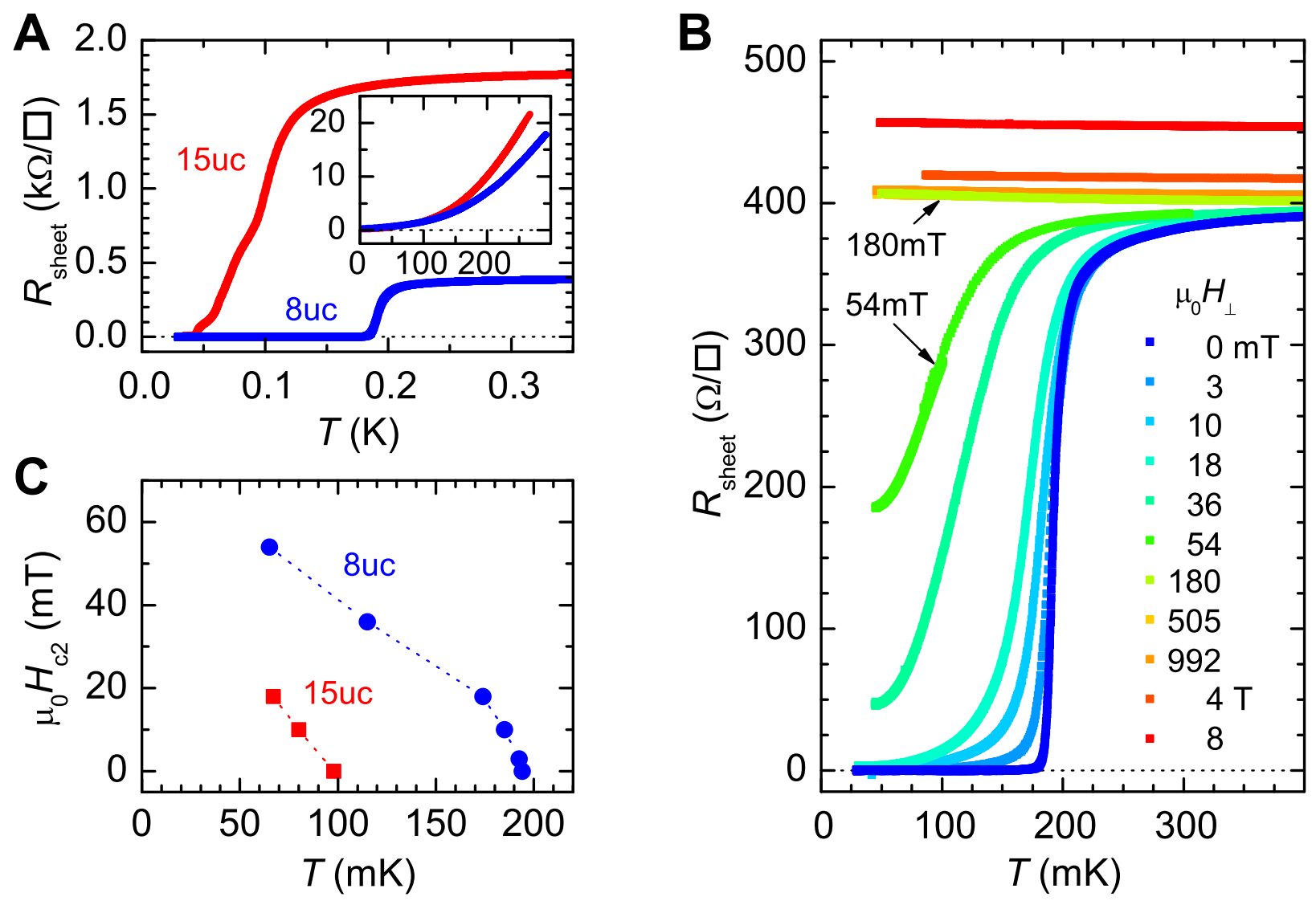

Fig. 2. Transport measurements on $\mathrm{LaAlO}_{3} / \mathrm{SrTiO}_{3}$ heterostructures. (A) Dependence of the sheet resistance on $T$ of the $8 \mathrm{uc}$ and $15 \mathrm{uc}$ samples (measured with a $100 \mathrm{nA}$ bias current). Inset: sheet resistance versus temperature measured between $4 \mathrm{~K}$ and $300 \mathrm{~K}$. (B) Sheet resistance of the 8 uc sample plotted as a function of $T$ for magnetic fields applied perpendicular to the interface. (C) Temperature dependence of the upper critical field $H_{\mathrm{c} 2}$ of the two samples. 

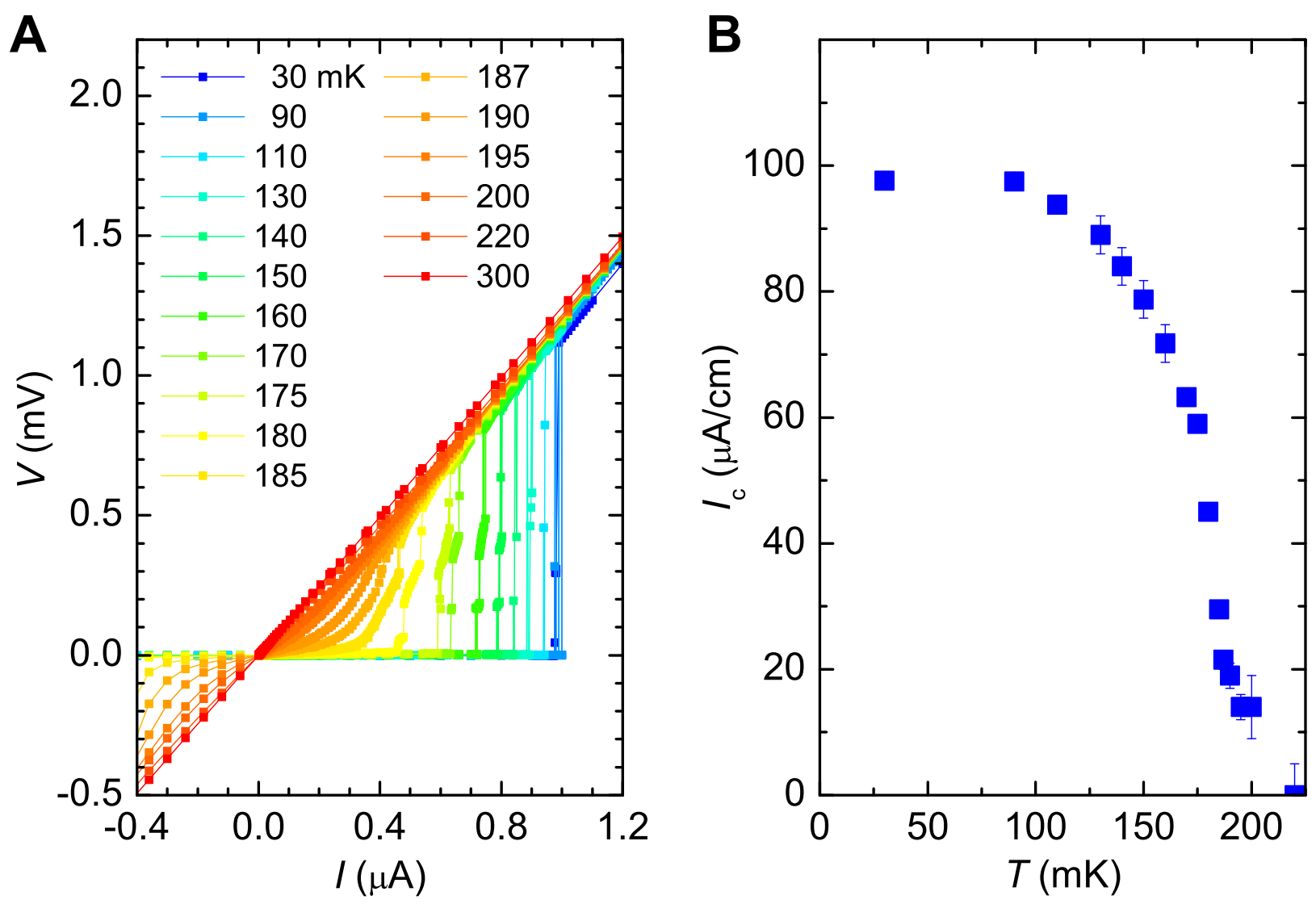

Fig. 3. $V(I)$ measurements of the $8 \mathrm{uc} \mathrm{LaAlO}_{3} / \mathrm{SrTiO}_{3}$ heterostructure. (A) Temperaturedependent current-voltage characteristics of a $100 \times 300 \mu \mathrm{m}^{2}$ bridge. (B) Measured temperature dependence of the linear critical current density, as obtained from (A). 

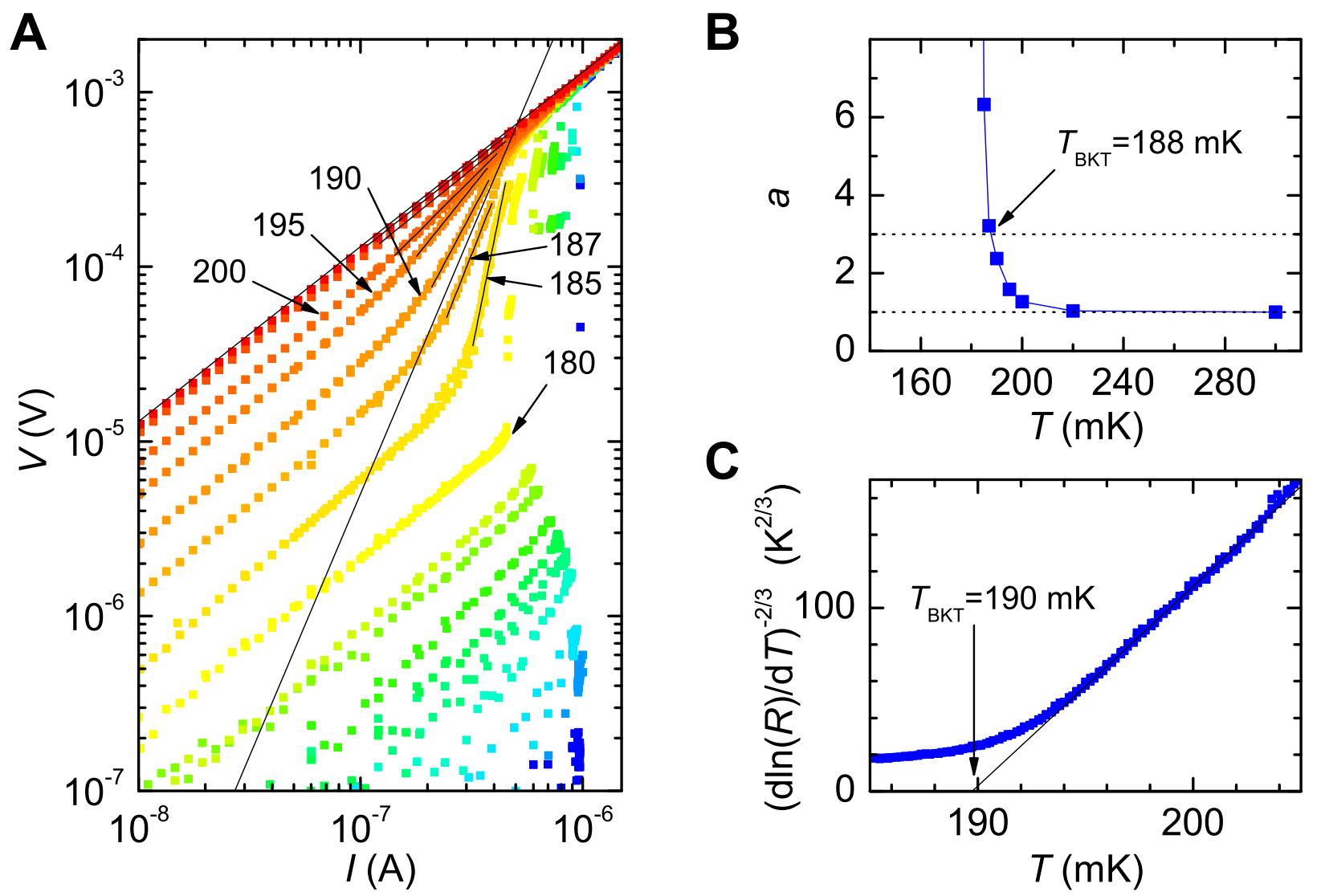

Fig. 4. Low temperature transport properties of the $8 \mathrm{uc} \mathrm{LaAlO}_{3} / \mathrm{SrTiO}_{3}$ heterostructure. (A) $V(I)$ curves on a logarithmic scale, the color code of Fig. 3A is used. The numbers provide the value of $T$, measured in $\mathrm{mK}$, at which the curves were taken. The short black lines are fits of the data in the transition. The two long black lines correspond to $V=R I$ and $V \sim I^{3}$ dependences and show that $187 \mathrm{mK}<T_{\mathrm{BKT}}<190 \mathrm{mK}$. (B) Temperature dependence of the power-law exponent $a$, as deduced from the fits shown in (A). (C) $R(T)$ dependence of the 8 uc sample $(I=100 \mu \mathrm{A})$, plotted on a $(\mathrm{d} \ln (R) / \mathrm{d} T)^{-2 / 3}$ scale. The solid line is the behavior expected for a BKT transition with $T_{\mathrm{BKT}}=190 \mathrm{mK}$. 


\section{Supporting Online Material}

\section{Materials and Methods}

\section{Transport Measurements:}

Transport measurements were done in a dc set-up on samples patterned into regular four-point line structures.

The perpendicular magnetic field is cancelled by minimizing the resistance as a function of the current in the magnetic coil with a precision leading to a residual field lower than $0.1 \mathrm{mT}$. This procedure is done at the temperature of the sharp superconducting transition step where the resistance is very sensitive to the magnetic field.

The voltage resolution is limited by thermal noise, the amplitude of which is mainly determined by the contact resistances. The measurements on the zero-voltage state were done by using a small current density to stay below the critical current.

\section{Electron Microscopy:}

The electron microscopy and spectroscopy measurements were performed on a monochromated $200 \mathrm{kV}$ FEI Tecnai F20-ST STEM with a minimum probe size of $\approx 1.6 \AA$ and a convergence semiangle of $(10 \pm 1) \mathrm{mrad}$. The HAADF image was recorded at an inner detector angle of $\approx 65 \mathrm{mrad}$. To increase signal to noise and average out the scan noise, 8 successive images, each recorded at 16 microseconds per pixel, were cross-correlated and averaged. Subsequently, the $1024 \times 1024$ pixel image was rebinned to $512 \times 512$ pixels.

For the EELS measurements, the Tecnai F20-ST is equipped with a Gatan imaging filter 865 -ER. The energy resolution was $\approx 0.6 \mathrm{eV}$ as measured from the FWHM of the zero loss peak at $0.2 \mathrm{eV} /$ channel. The Ti- $\mathrm{L}_{2,3}$ and $\mathrm{O}-\mathrm{K}$ spectra were recorded simultaneously on a $2048 \times 2048$ pixel CCD. To increase signal to noise 5 successive spectra, each recorded for $5 \mathrm{~s}$, were averaged. 